\title{
What can big data tell us about past, current, and future patterns of infection?
}

by Ann Seongju Yun, Maliha Islam, Samee Mahbub, Aanal Mehta, Dennis Windsor, Monica Patel, Nashwan Abdulhamid, Xing Hu, Kaiqi Cheng, Geumhui Han, Rebecca Do, Yasha Farhan, Mikerah Quintyne-Collins, Jessie Zheng, Adam Skudlarski, Delaram Dara, Kevin Faust, Michael K. Lee, Bobak Mahtash, Arushi Sachdev and Kerman Sekhon.

STEM Fellowship's Big Data Challenge is a unique pedagogical experiment, providing an inquiry and learning experience for high school students that, upon equipping them with top-notch analytical tools, tasks them to find hidden patterns and trends in complex socioeconomic or scientific data. In May 2017, this challenge format was applied for the first time at the undergraduate level, providing teams of university students with the opportunity to delve into datasets related to public health and epidemiology in order to make useful inferences about health and disease.

Published here are the abstracts from all entrants. Teams employed a variety of approaches in their respective projects, whether to look at disease from a strictly socioeconomic angle or to examine the efficacy of preventative measures. For all the variation between project themes, it remains that all submissions are of incredibly high quality. Every paper is demonstrative of immense creativity and high potential on the respective team's part.

On behalf of STEM Fellowship, I would like to extend my heartfelt congratulations to all students who participated in the challenge, and I wish them all the best for their future endeavours in research and data science. It has been a privilege for us to witness the analytical capabilities of the next generation of students firsthand, and I am certain all entrants will only continue to demonstrate excellence in their respective research careers.

\section{Ahmed Hasan \\ Director for Data Science Education, STEM Fellowship Big Data Challenge 2016-2017 Team Lead}

\section{Disclaimer}

These abstracts are provided for all student teams that have submitted project reports by May 30, 2017. The STEM Fellowship Journal's editorial board has made every effort to ensure proof and editing of these abstracts in a limited amount of time, and neither organization as a whole or any of its volunteer members can be held accountable for inaccuracies that may have occurred in the abstract publication. Abstracts are published in alphabetical order per names of the participating teams.

\section{Developing a Syndemic Framework for Understanding HIV Prevalence}

\section{Ann Seongju Yun, Maliha Islam, and Samee Mahbub}

\section{Abstract}

This paper aims to analyze the HIV prevalence of different countries based on various markers, such as economic health and environmental status. Despite existing studies that show how these factors affect and correlate with HIV prevalence, they are mostly done independently. The inter-correlations between these factors and how they affect the HIV prevalence have rarely been considered. Datasets were collected from organizations such as the World Health Organization (WHO), the World Bank, and UNESCO, analyzed using software tools from R, such as K-means clustering and linear regression, and then visualized using R's libraries. Statistical tools were used to interpret these datasets, and to accept or reject the hypotheses that were originally constructed (or left inconclusive if the correlation is not strong enough). Afterwards, these individual factors were then correlated in an attempt to understand how they affect the HIV prevalence of a given country using a syndemic framework. The datasets indicated that economic factors, such as the gross domestic product (GDP) and the Gini coefficient, were strongly correlated to HIV prevalence. Specifically, GDP showed a negative correlation, while the Gini coefficient showed a posi- 
tive correlation. Other trends were also found in markers, such as the Climate Change Performance Index (CCPI), demonstrating that most countries had mid to low values of CCPI. Furthermore, data based on various response methods to HIV prevention, such as antiretroviral therapy and HIV testing and counselling, were shown to have contradictory correlations. HIV testing and counselling was found to have a positive correlation with HIV prevalence, and this was very contradictory as a negative correlation was expected. Lastly, a syndemic framework was constructed using correlational research between the factors themselves, to understand how different factors interact synergistically to increase the prevalence of HIV, and to more accurately predict HIV prevalence trends.

\section{Insecticide usage, resistance, and their effect on malaria}

Aanal Mehta, Dennis Windsor, Monica Patel, and Nashwan Abdulhamid

\section{Abstract}

Malaria is a life-threatening disease caused by the females of the "malaria vector" Anopheles mosquito sub-species. Once the females bite humans, the parasites are passed via the bloodstream where they continue to grow and asexually multiply. In severe cases, malaria can cause seizures, comas, and death. With the rising levels of estimated malaria cases, particularly in Sub-SaharanAfrica, it is imperative to analyze the more prominent causes of this epidemic. It was hypothesized that the increase in malaria cases, especially in Sub-Saharan Africa, was due to an increase in insecticide resistance of malaria vectors, which stemmed from an upsurge in insecticide usage of the respective countries. Three variables were tested in this study: insecticide usage, resistance, and estimated malaria cases. The data was imported into a Jupyter notebook running Python 2.7 and sorted into two separate scales: a global scale and a Sub-Saharan African scale. Then, Pearson correlation tests were performed to test the correlations between the three variables and to graph the regressions. The results did not support the hypothesis, as there was no positive correlation between insecticide usage and malaria cases. After failing to reject the null hypothesis, an alternative conclusion was reached, as a portion of the initial hypothesis was supported. The data showed a weak positive correlation between insecticide resistance and malaria cases on a global scale. Therefore, it can be predicted that decreasing the number of insecticide-resistant malaria vectors will result in a decrease in malaria cases. As such, the policies proposed were catered towards this alternative hypothesis, with an emphasis placed on reducing resistant vector populations and preventing infections. Currently, the datasets are incomplete in various areas, as such results are skewed and can negatively affect the correlational results being studied. If country-wide data is further split into regional data, and insecticide resistance data is presented in a non-binary format, the expected positive correlations should be observed. With extensive care put towards social and political policies, such as water regulation, microorganism usage, and chemical usage, projected decreases in annual malaria cases should be seen.

\section{A Look into Factors Relating to HIV Cases on a Country-by-Country Basis}

Xing Hu, Kaiqi Cheng, Geumhui Han, and Rebecca Do

\section{Abstract}

This study aims to find the inner relationships between 12 variables (GDP, population, region, number of new infections, number of deaths of HIV, number of SW, number of MSM, number of IDU, number of pregARV, number of uses of ART, laws about HIV) and their effects on the number of patients living with HIV. The analyses are based on the dataset that covers 133 countries. The multiple linear regression shows that some factors have interactive effects on the number of people living with HIV, which suggests a potentially efficient way to decrease HIV cases by increasing or decreasing the number of interacting variables at the same time. A logistical regression model was also built to predict potential HIV cases in a country based on other factors.

\section{How the Immunization Of Youth Influences the Mor- tality Rates Of Tuberculosis Around The World}

Yasha Farhan, Mikerah Quintyne-Collins, Jessie Zheng, and Adam Skudlarski

\section{Abstract}

Millions of individuals around the world are currently living with Tuberculosis (TB), and while there have been many efforts to eliminate its spread, the TB epidemic will not be eradicated anytime soon. However, timely administration of vaccines to the youth for diseases other than TB (such as Measles, Polio and Tetanus) may bring us one step closer to the eradication of it. Correlations be- 
tween multiple vaccinations of the youth, TB mortality rates, and the deaths of children under five years old were obtained. The resulting data implied that as the number of one-year-old children getting vaccinated for diseases other than TB increases, the mortality rate of TB decreases. Continuing this decrease in TB mortality rates is the key to eradicating TB, the disease that kills millions, but silently affects millions more.

\section{Worldwide Population Study on Patterns of Human Immunodeficiency Virus (HIV) and Tuberculosis (TB) Cases}

Delaram Dara, Kevin Faust, Michael K. Lee, Bobak Mahtash

\section{Abstract}

It has been previously established that Human Immunodeficiency Virus (HIV) is a major risk factor for tuberculosis (TB). In this paper, we took a multidisciplinary approach of combining the fields of bacteriology, virology, and epidemiology. We assessed the consequences of having both HIV and tuberculosis using datasets that document the worldwide occurrence of TB and HIV, as well as graphical representations and various statistical analyses through Python 3.5 programming. Using this epidemiological approach, we then hypothesized that HIV increases the chance of an individual contracting both pulmonary (TB) and extrapulmonary TB (EPTB). Furthermore, socio-economic consequences of HIV and TB coinfection were evaluated by considering other risk factors that increase the likelihood of acquiring TB. As a final note, we provide some future studies and improvements that can be pondered upon for upcoming papers in this field.

\section{Potential Predictors for HPV Vaccination Completion Rates}

\section{Arushi Sachdev and Kerman Sekhon}

\section{Abstract}

Human papillomavirus (HPV) is an infectious agent responsible for a common sexually transmitted infection. The virus is so easily transmitted to the point where most men and women have been, or will be infected at some point in their lifetime. While the majority of infections are asymptomatic or can be resolved on their own, there are a subset of strains that cause genital warts, cervical cancer, and various anogenital cancers. Given the virus' widespread dissemination in the population, it is no surprise that cervical cancer is the second leading cancer to cause death in females. Accordingly, a vaccine has been made to prevent HPV infection, and in turn, prevent the onset and development of cervical cancer. While the vaccine, Gardasil, is effective against over $75 \%$ of cervical cancers, recent studies have demonstrated its limited adoption. Moreover, Gardasil is a three-dose vaccine, and consequently, the female patients that do initiate the vaccination series often do not complete it in its entirety. To substantiate these claims, data obtained from researchers at the Johns Hopkins Medical Institutions were used to determine which socioeconomic factors influence a female's likelihood of vaccination completion. Specifically, this study performed a logistic regression and LASSO logistic regression. Findings revealed that certain female cohorts are at risk of incomplete vaccinations, such as Black females, females between the ages of 18-26, and females that visited urban clinics. In contrast, females that were treated by obstetrician-gynecologists had increased compliance with the Gardasil vaccination regimen. These conclusions are not causal, however, they shed light on the female populations that should be studied further and potentially targeted to improve Gardasil vaccination completion rates. 